\title{
Análisis Estadístico Matemático del Comportamiento de Las Condiciones Eléctricas Establecidas en los Ensayos de la Norma ISO 16750-2 en Dos Módulos de Alarma Automotriz en el Ecuador
}

\author{
Pancha, Johnny ${ }^{1}$; Rojas, Vicente ${ }^{1}$; Lema, Jorge ${ }^{2}$; Arteaga, Gerardo ${ }^{2}$ \\ ${ }^{1}$ Escuela Superior Politécnica de Chimborazo, Facultad de Mecánica, Riobamba, Ecuador \\ ${ }^{2}$ Universidad Tecnológica Indoamérica, Facultad de Ingeniería y Tecnologías de la Información y Comunicación, Quito, \\ Ecuador
}

\begin{abstract}
Resumen: Los dispositivos electrónicos que han sido diseñados para ser instalados en el vehículo pueden ser sometidos a pruebas necesarias para obtener una certificación o aprobación mínima requerida para ser instalada en el sistema eléctrico del automóvil. Con el uso de un equipo normado el cual permite generar condiciones eléctricas a un dispositivo electrónico, se han obtenido diferentes resultados ante el comportamiento eléctrico que se puede encontrar especialmente en el vehículo; condiciones tales como: caídas momentáneas de tensión, sobretensiones e inversiones de tensión de voltaje, considerando incluso someter los dispositivos a las fuertes condiciones eléctricas del sistema automotriz cuando se produce el arranque del motor. Con este análisis se obtiene resultados del comportamiento entre dos dispositivos electrónicos y de tal manera determinar mediante la validación de cada uno, si las características del diseño o aspectos de programación pueden influir en el resultado de cada prueba. Esto nos permite considerar varios aspectos ante el diseño del dispositivo y de esta manera lograr conseguir un óptimo funcionamiento; siendo esto reflejado en obtener una certificación.
\end{abstract}

Palabras clave: Módulos de alarma; ISO 16750-2; Condiciones Eléctricas, equipo chroma.

\section{Mathematical Statistical Analysis About the behavior of the Electrical Conditions Established under the ISO Standard Test 16750-2 in Two Automotive Alarm Modules in Ecuador}

\begin{abstract}
Electronic devices that are designed to be installed in the vehicle may be subject to tests required for certification or minimum approval required to be installed in the electrical system of the car. With the use of a standardized equipment which allows to generate electrical condition to an electronic device, to obtain different results before the electrical behavior that can be found especially in the vehicle; conditions such as: momentary voltage falls, surge voltage and invertion voltage, whereas even submit devices to automotive system strong electrical conditions when starting the engine. With this analysis, results of the behavior between two electronic devices are obtained and in this way it is determined through the validation of each one, if the characteristics of the design or aspects of programming can influence the result of each test. This allows us to consider several aspects of the design of the device and thus achieve optimum performance, this being reflected in obtaining a certification.
\end{abstract}

Keywords: Alarm modules; ISO 16750-2; Electrical Conditions, chroma equipment.

\section{INTRODUCCIÓN}

La norma ISO $16750-2$ proporciona todas las pruebas a ejecutarse en un dispositivo electrónico que está diseñado para trabajar en el vehículo y acoplarse de manera ideal al sistema eléctrico del mismo. Por lo tanto, algunas marcas automotrices reconocidas en el mercado nacional han adaptado ciertas pruebas detalladas en dicha norma para garantizar calidad en los módulos electrónicos que son ensamblados en el vehículo, como pueden ser: radios, módulos de alarma, módulos de gestión electrónica automotriz y sensores, etc. Durante la ejecución de estas pruebas se obtienen algunos resultados relacionados a la tensión de voltaje y variaciones en sus frecuencias; que son analizados en este artículo para determinar ciertas condiciones en las que los dispositivos electrónicos presentan diferenciaciones en su funcionamiento.

Específicamente los elementos sometidos a análisis son dos módulos de alarmas automotrices, que al ejecutar las pruebas detalladas en la norma se han obtenido resultados diferentes, lo que ha permitido establecer ciertos parámetros que según nuestro criterio influyen en el comportamiento de diseño o programación del módulo. La obtención de resultados surgen de la aplicación de las siguientes pruebas específica en la norma: Caída momentánea de tensión, condición de arranque 
en la cual se clasifica en 4 diferentes pruebas, prueba de sobretensión y prueba de inversión de tensión.

\section{MARCO TEÓRICO/METODOLOGÍA}

\subsection{Alarma Automotriz}

Para el análisis del comportamiento de un dispositivo electrónico en las condiciones eléctricas que pueden desarrollarse en el vehículo, se ha considerado a la alarma automotriz, siendo éste un dispositivo instalado en la gran mayoría de vehículos, cuyo funcionamiento debe ser óptimo debido a que si llegase a presentar fallas debido a problemas en el sistema eléctrico coloca en peligro la protección del automotor ante un robo, es decir lo puede volver vulnerable en ese tipo de situaciones (Alonso J., 2014).

La alarma automotriz, tiene muchas funciones básicas que son programadas en la unidad de control, las cuales no deben sufrir daños en su software, tales como desconfiguraciones o saltos en su programación, (Antoni J., 2011). Para el análisis se ha seleccionado como función, la condición de anti asalto, en donde el modulo electrónico está programado para cumplir dos acciones importante; corte al motor, es decir, elimina la alimentación de energía a la bobina de encendido o la bomba de combustible para inmovilizar al vehículo y evitar que salte de esta condición con el uso del control a distancia de la alarma, y éste únicamente pueda ser desactivado mediante un código pin a través de un botón valet, (Antoni J., 2011). Al realizar las pruebas determinadas en este análisis, se ha comprobado el comportamiento de dos diferentes módulos de alarmas en las pruebas determinadas en la ISO 16750-2.

\subsection{Equipo}

\section{Hardware}

Para la realización de las pruebas se usa un equipo normado, el cual es constituido por dos aspectos, la parte de hardware, elemento que envía la tensión hacia los módulos de alarma; la configuración de los valores de tensión y amperaje, puede realizarse desde un programa instalado en un ordenador o directamente a través del panel de teclado del equipo. Posee 4 salidas de corriente ( 2 positivas y 2 negativas) y una pantalla LCD donde se puede visualizar el voltaje y amperaje en tiempo real (Alonso J., 2014) en la Figura 1 se muestra el hardware del simulador.

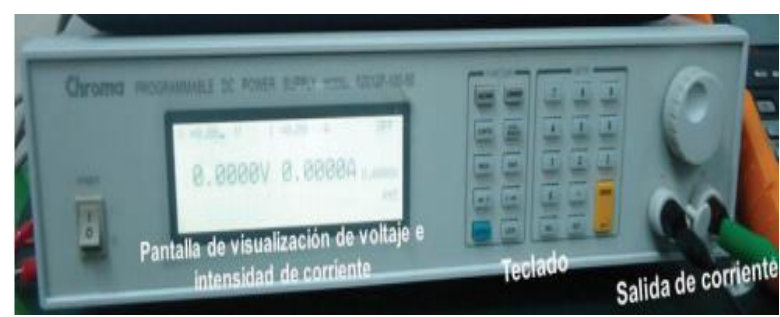

Figura 1. Equipo de simulación DC Power Supplied Chroma

\section{Software}

Para la operación y funcionalidad del equipo se requiere de la aplicación de un software que es el encargado de manejar y controlar al equipo para realizar las pruebas que se le designen, es decir, aquí se puede seleccionar las pruebas establecidas en la norma, como también realizar pruebas personalizadas según los requerimiento,(Alonso J., 2014). En la Figura 2 se tiene una imagen detallada de la interfaz del programa.

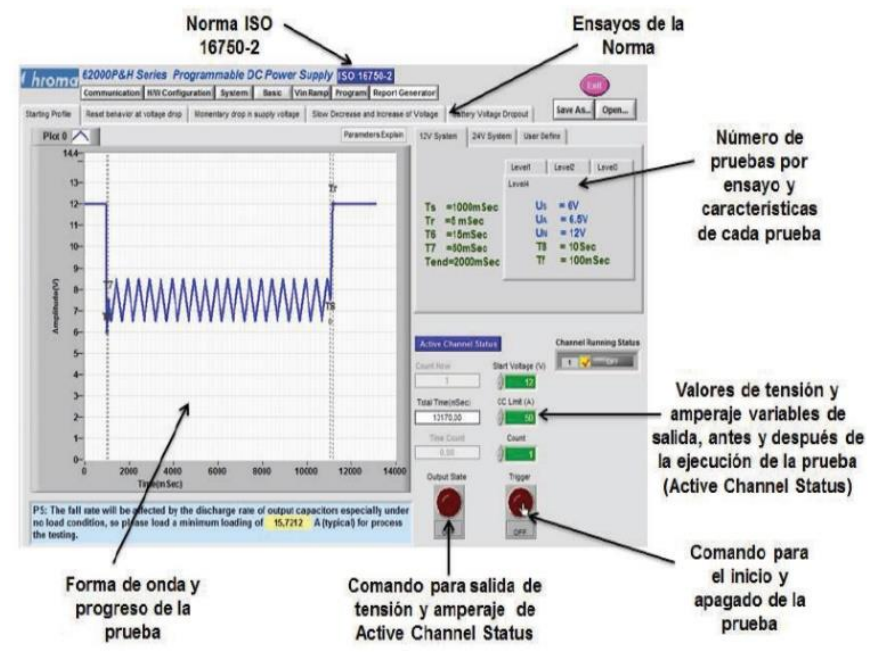

Figura 2. Interfaz del manejo del equipo de ensayo

\subsection{Ensayos}

En primera instancia, es necesario tener claro cómo se aplica la norma; determinar cuáles son las condiciones de las pruebas, aspectos iniciales o previos durante la ejecución de cada una de ellas y poder especificar qué ponderación obtuvo el dispositivo electrónico después del ensayo. Estos aspectos están detallados en la norma ISO 16750-1,(Daniels J., 2010). La información detallada de cada una de las pruebas, de los parámetro a tomarse en cuenta antes, durante y después de la prueba están especificados en la norma ISO 16750-2,(Lema J., 2016).

De ahí la importancia de tener una lectura clara y amplia para poder adquirir resultados específicos. Las pruebas que se explica a continuación pueden llegar a ser destructivas dependiendo del diseño y programación del módulo de alarma, en otros casos simplemente será una evaluación de rutina de los módulos.

El uso del equipo adecuado es primordial, para la evaluación se utiliza un equipo que facilita la ejecución de las pruebas; se optó para este fin utilizar un equipo marca Chroma.

\section{Voltaje mínimo}

Como punto inicial de las pruebas se requiere someter a los módulos electrónico, y por tanto conocer el voltaje mínimo de funcionamiento tal como lo detalla la norma ISO 16750-1, lo que determina el voltaje mínimo de funcionamiento para cada módulo de alarma,(Daniels J., 2010).

Se inicia el proceso de la evaluación de cada dispositivo; para lo cual cada prueba se ha establecido con base a un protocolo de revisión el cual se realiza antes y después de la prueba para registrar si existe alguna anomalía o falla en el dispositivo, de esta manera se consigue establecer un procedimiento formal 
para cada una de las pruebas. Es inherente mencionar que cada valor tiene una tolerancia de +/- 0.2 voltios. En la Tabla 1 se observa la clasificación designada en la norma ISO 16750-1 sobre el voltaje mínimo de funcionamiento.

Tabla 1. Clasificación de equipo en función del voltaje mínimo de

\begin{tabular}{ccc}
\multicolumn{3}{c}{ funcionamiento } \\
\hline Código & $\begin{array}{c}\text { Voltaje alimentación }(\mathbf{V}) \\
\text { Usmin }\end{array}$ & $\begin{array}{cc}\text { Usmax } \\
\end{array}$ \\
\hline A & 6 & 16 \\
B & 8 & 16 \\
C & 9 & 16 \\
D & 10.5 & 16 \\
\hline
\end{tabular}

\section{Ensayo caída momentánea de tensión}

Esta prueba somete al equipo a una condición en donde la alimentación principal de tensión sufre una variación, la cual hace disminuir la tensión durante una fracción de segundo para luego regresar al valor alimentación de tensión inicial, simulando una posible falla cuando existe algún tipo de contacto entre otro cable de conexión o una conexión de tierra deficiente,(Lema J., 2016). En Figura 3 se observa la característica de la señal de la prueba relacionando el voltaje en función del tiempo.

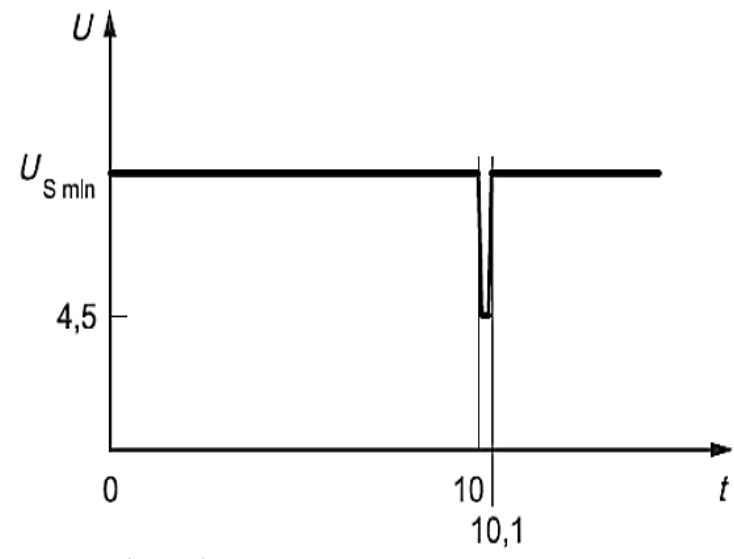

Figura 3. Ensayo caída momentánea de tensión

\section{Ensayo reseteo ante la caída de tensión}

El ensayo establecido en esta prueba consiste en ir descendiendo los valores de tensión en un 5\% del valor inicial, esta prueba se relaciona con la caída momentánea de tensión con la diferencia que se ejecuta varias veces hasta llegar a un valor de tensión 0 . El tiempo determinado se estima en 5 segundos en donde se mantiene la caída de tensión y 10 segundos en el valor inicial de tensión,(Lema J., 2016). En la Figura 5 se presenta la gráfica del ensayo considerando el voltaje en función del tiempo.

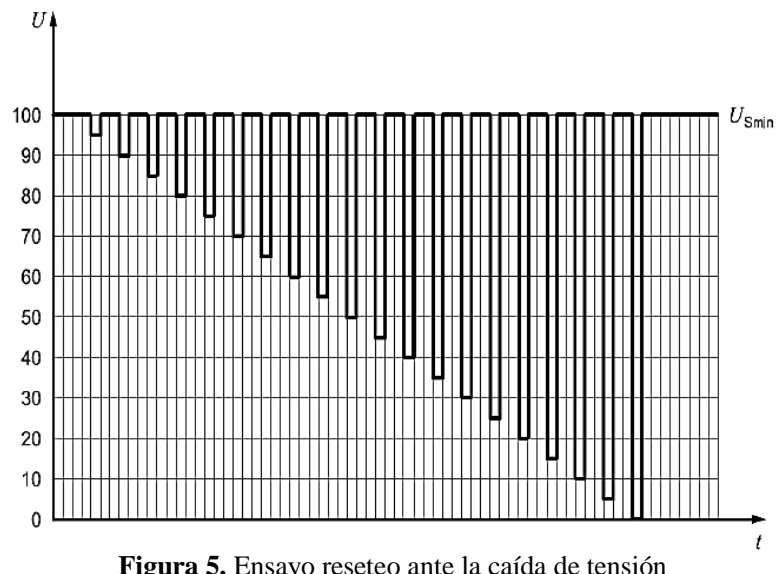

\section{Ensayo condición de arranque}

El sistema eléctrico del vehículo está sometido a condiciones muy particulares cuando se actúa el motor de arranque para producir los giros iniciales al motor para que éste pueda encenderse; la condición de arranque es un gran desafío para un dispositivo electrónico instalado en el automóvil. En la ejecución de la prueba se debe cumplir la repetición de 10 veces de cada una de la pruebas en sus 4 niveles que conforma este ensayo,(Lema J., 2016). En la Figura 4 se observa el voltaje en función del tiempo, al realizar la prueba de arranque.

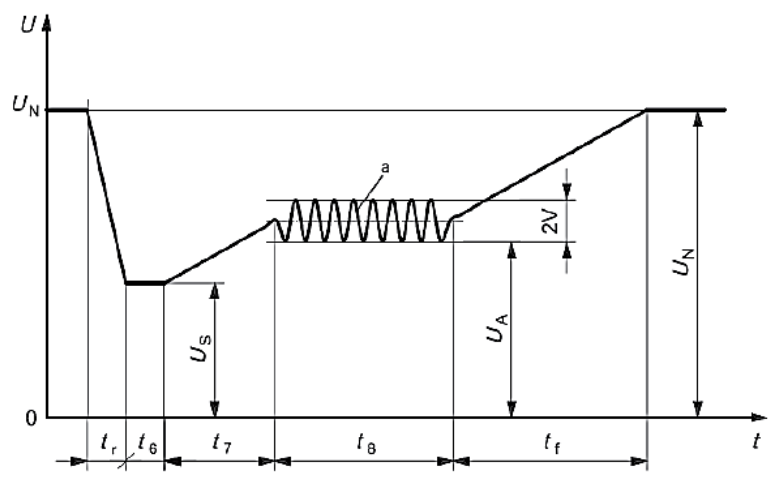

Figura 4. Condición de arranque

En el ensayo de arranque es importante considerar que la norma ISO 16750-2 determina que existen 4 niveles; es decir, se debe ejecutar estas pruebas que son distintas, en la Tabla 2 se muestra los valores de tensión en el tiempo especificado para cada nivel de la prueba de arranque.

Tabla 2. Ensayo de arranque en sus 4 niveles diferentes

\begin{tabular}{|c|c|c|c|c|}
\hline VARIABLES & $\begin{array}{l}\text { PRUEBA } \\
\text { NIVEL } 1 \\
\end{array}$ & $\begin{array}{l}\text { PRUEBA } \\
\text { NIVEL } 2 \\
\end{array}$ & $\begin{array}{l}\text { PRUEBA } \\
\text { NIVEL } 3 \\
\end{array}$ & $\begin{array}{l}\text { PRUEBA } \\
\text { NIVEL } 4 \\
\end{array}$ \\
\hline $\mathbf{U}_{\mathbf{S}}$ & $8 \mathrm{~V}$ & $4.5 \mathrm{~V}$ & $3 \mathrm{~V}$ & $6 \mathrm{~V}$ \\
\hline $\mathbf{U}_{\mathbf{A}}$ & $9.5 \mathrm{~V}$ & $6.5 \mathrm{~V}$ & $5 \mathrm{~V}$ & $6.5 \mathrm{~V}$ \\
\hline $\mathbf{U}_{\mathrm{N}}$ & $12 \mathrm{~V}$ & $12 \mathrm{~V}$ & $12 \mathrm{~V}$ & $12 \mathrm{~V}$ \\
\hline$t_{r}$ & $5 \mathrm{~ms}$ & $5 \mathrm{~ms}$ & $5 \mathrm{~ms}$ & $5 \mathrm{~ms}$ \\
\hline$t_{6}$ & $15 \mathrm{~ms}$ & $15 \mathrm{~ms}$ & $15 \mathrm{~ms}$ & $15 \mathrm{~ms}$ \\
\hline $\mathbf{t}_{7}$ & $50 \mathrm{~ms}$ & $50 \mathrm{~ms}$ & $50 \mathrm{~ms}$ & $50 \mathrm{~ms}$ \\
\hline $\mathbf{t}_{8}$ & $1 \mathrm{~s}$ & $10 \mathrm{~s}$ & $1 \mathrm{~s}$ & $10 \mathrm{~ms}$ \\
\hline $\mathbf{t}_{\mathbf{f}}$ & $40 \mathrm{~ms}$ & $100 \mathrm{~ms}$ & $100 \mathrm{~ms}$ & $100 \mathrm{~ms}$ \\
\hline
\end{tabular}




\section{RESULTADOS Y DISCUSIÓN}

\subsection{Análisis matemático y estadístico}

Los resultados obtenidos con base a la captación de señales y datos en las pruebas de ambas alarmas permitieron realizar modelos de simulación matemática para identificar el comportamiento en modelos de ecuaciones.

La modelación se realizó con base al comportamiento de las señales, las cuales permiten un análisis estructural más detallado por la forma de dichos diagramas como se indicará en los siguientes apartados.

\section{Ensayo caída momentánea de tensión}

Como resultado en este ensayo se obtuvo que en un módulo de alarma, después de la ejecución de la prueba salió de la función de anti asalto de la alarma; mientras que otro módulo de alarma se mantuvo en la función programa antes de la ejecución de la prueba. En la Figura 6 se puede interpretar el resultado del comportamiento lineal en la prueba de discontinuidad, observando que en ambos tipos de alarma la caída es igual, y es en una fracción de décima de segundo, por lo cual el análisis matemático de comportamiento no es necesario.

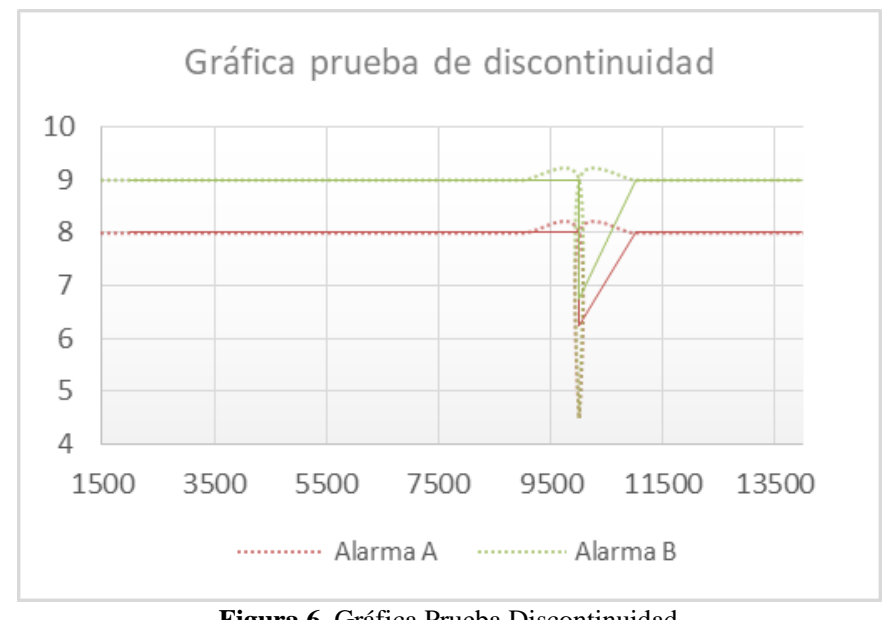

Figura 6. Gráfica Prueba Discontinuidad

El análisis para la regresión únicamente se necesita una media móvil debido a la complejidad de la gráfica; ya que se posee un conjunto de datos lineales cuyo análisis de modo de puntos se crea con base a un promedio entre un punto de salto, con esto se obtiene la media móvil de un subconjunto de datos originales.

\section{Ensayo reseteo ante la caída de tensión}

Los resultados obtenidos según la condición de mantenerse en la función de anti asalto que la alarma A salió de la función al inicio de la prueba, es decir, cuando la tensión bajo a 6 voltios; mientras que la alarma B se mantuvo en la función de anti asalto durante toda la ejecución y al finalizar la prueba. En la Figura 7 se observa el comportamiento lineal de la prueba generando en este caso con respecto a la prueba de discontinuidad un modelo matemático.

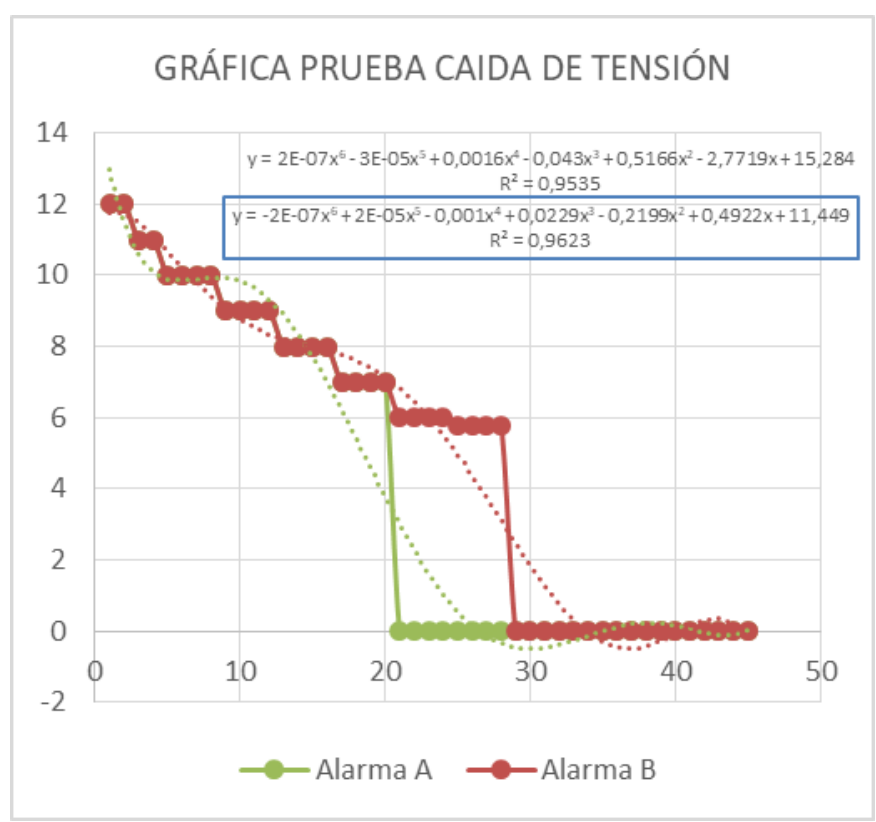

Figura 7. Gráfica Prueba Caída De Tensión

En la siguiente gráfica de modelación se realizó una regresión polinómica debido a la cantidad de datos y la dispersión de los mismo por lo cual no se utiliza un modelo matemático complejo ya que como se observa en las ecuaciones de resultado y el factor confiabilidad son de 95\% para ambos casos, indicando una aproximación optima la comportamiento de las pruebas, para asi interpretar el valor de error más bajo.

\section{Ensayo condición de arranque nivel 1}

En el ensayo de arranque nivel 1, la alarma A salió de la función de anti asalto al ejecutarse las pruebas, en el caso de la alarma B ésta mantuvo la función de anti asalto. Es importante mencionar que en las pruebas de arranque la alimentación a este tipo de módulos de alarma según la norma es de 12 voltios.

En la Figura 8 se puede verificar el comportamiento de la señal en base a la prueba de arranque en condiciones de nivel 1 de exigencia; generando el trazado del comportamiento y el análisis de la media móvil debido a que la misma es el promedio de comportamiento de una dispersión de datos adquiridos con base a una prueba, permitiendo un promedio del comportamiento.

El factor de error de la suma de cuadrados indica que los factores de cambio de curva en los puntos de giro son mas estables durante el proceso, aseverando un resultado más preciso durante toda prueba; con un valor inferior al $10 \%$ de error. Y una aproximación del $64.96 \%$, que aunque no supera el $85 \%$ de acercamiento, se puede verificar en la Figura 8 y el modelo matemático (Ec.1) un acoplamiento estable.

En las diferentes pruebas de arranque realizadas, por la forma del comportamiento que se vio desde la adquisición de las señales, se puede observar que su comportamiento se asemeja a las funciones trigonométricas, procedemos a realizar un ajuste por el método de suma de senos, ya que como se indica en los siguientes apartados da una veracidad mayor del $70 \%$ en la aproximación de dichas curvas. 
Para cada ecuación realizada, la variable de cambio y de control es el tiempo, en base a la variación de la tensión. La modelación se realizó en el software Matlab.

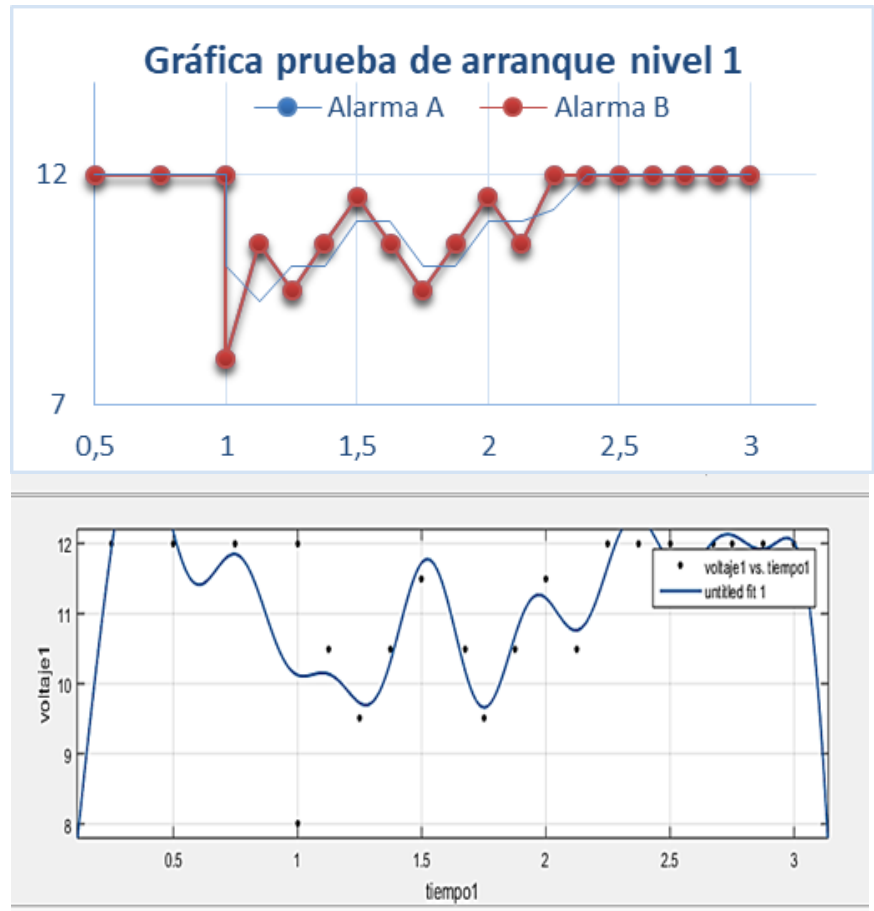

Figura 8. Gráfica prueba de arranque nivel 1

$$
\begin{aligned}
f(t)=a 1 * \sin & (b 1 * t+c 1)+a 2 * \sin (b 2 * t \\
& +c 2)+a 3 * \sin (b 3 * t+c 3) \\
& +a 4 * \sin (b 4 * t+c 4)+a 5 \\
& * \sin (b 5 * t+c 5)+a 6 * \sin (b 6 \\
& * t+c 6)+a 7 * \sin (b 7 * t \\
& +c 7)
\end{aligned}
$$

\begin{tabular}{|c|c|c|c|c|c|c|c|}
\hline $\begin{array}{c}\text { Coeficient } \\
\text { e }\end{array}$ & 1 & 2 & 3 & 4 & 5 & 6 & 7 \\
\hline & 19.2 & 12.7 & 14.4 & 15.0 & 0.39 & 5.07 & 0.52 \\
\hline $\mathbf{a}$ & 9 & 3 & 4 & 9 & 5 & 9 & 4 \\
\hline b & $\begin{array}{c}1.37 \\
2\end{array}$ & 2.77 & 5.05 & 5.77 & 11.7 & $\begin{array}{c}6.66 \\
3\end{array}$ & $\begin{array}{c}15.9 \\
1\end{array}$ \\
\hline c & -0.63 & 0.31 & -0.18 & 1.83 & $\begin{array}{c}- \\
2.98 \\
7\end{array}$ & $\begin{array}{c}3.66 \\
9\end{array}$ & $\begin{array}{c}2.20 \\
5\end{array}$ \\
\hline
\end{tabular}

Valores de ajuste: SSE: $8.993, \mathbf{R}^{2}: 0.6496=64.96 \%$

Tabla 3. Coeficientes reemplazo ecuación prueba arranque 1

\section{Ensayo condición de arranque nivel 2}

Los resultados de la prueba de arranque nivel 2 mostraron que la alarma A no se mantiene en la función de anti asalto mientras al ejecutarse la prueba, al contrario lo que sucede con la alarma B se mantiene en la función de anti asalto. En la Figura 9 se ve el comportamiento en diente de sierra para lo que se utiliza un modelo de suma de senos ya que el de Fourier es una aproximación inferior.

El factor de error de la suma de cuadrados indica que los factores de cambio de curva en los puntos de giro son más estables durante el proceso de desarrollo, con un factor de error de cuadrados mayor que es de $35.88 \%$ y una aproximación de
$77.37 \%$, generan un comportamiento del proceso en si es mucho más estable, generando ese aumento error por el cambio de tensión del módulo tanto en arranque como final, pero un enlace más estable en su proceso de prueba, lo que podemos verificar en la Figura 9 y el modelo matemático (Ec.2) un acoplamiento estable.

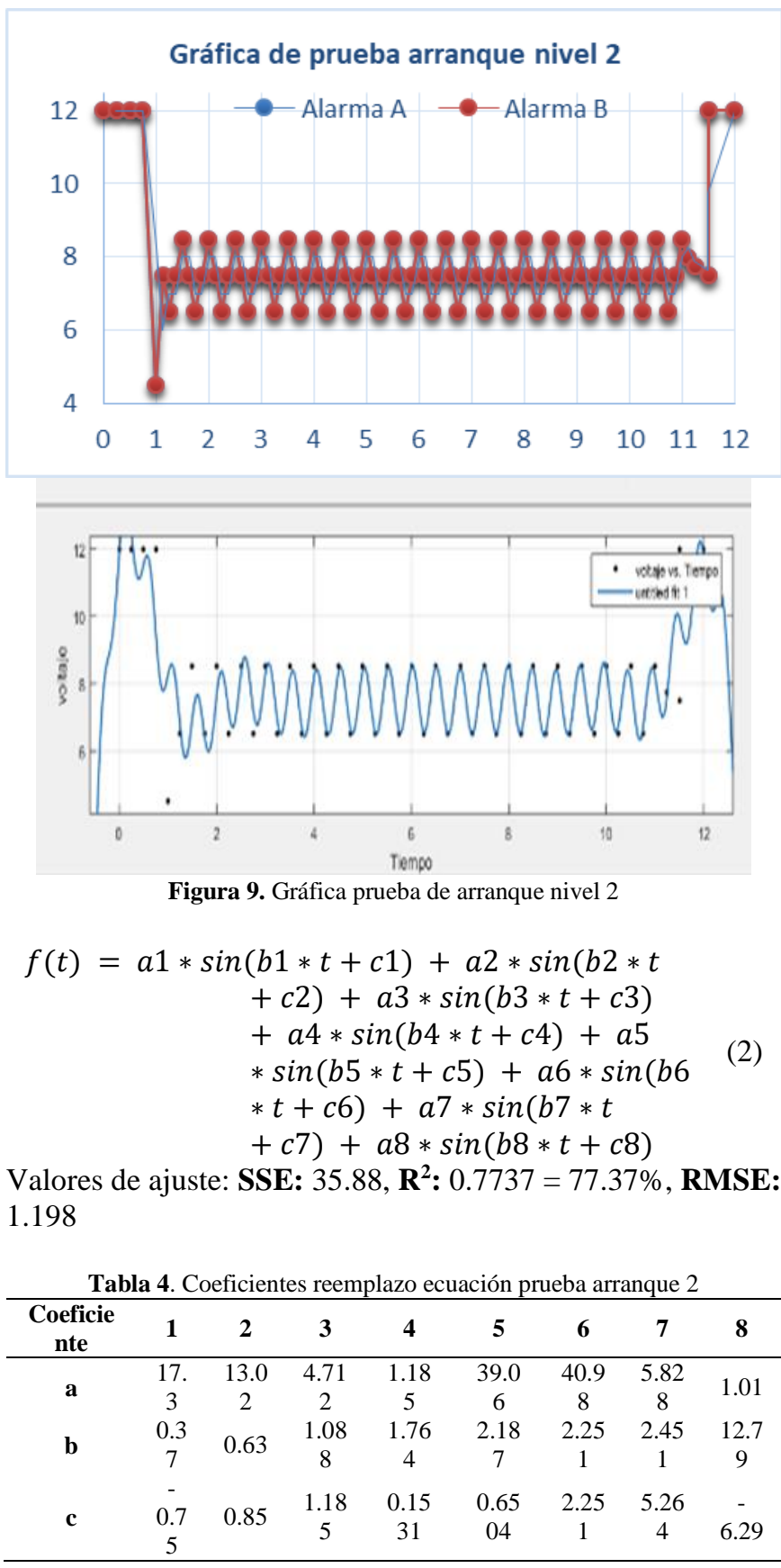

\section{Ensayo condición de arranque nivel 3}

Como resultado de esta prueba se obtiene que la alarma A sale de la función de anti asalto durante la prueba, mientras que en el caso de la Alarma B, ésta se mantiene en dicha función durante y al finalizar la prueba. En la Figura 10 se ve el comportamiento de la señal y los picos de descarga.

El factor de error de la suma de cuadrados para la condición 3 de arranque indica que los factores de cambio de curva en los 
puntos de giro son más estables durante el proceso de desarrollo, con un factor de error de cuadrados mayor que es de $42.06 \%$ y una aproximación de $77.71 \%$, generan un comportamiento del proceso inestable, generando ese aumento error por el cambio de tensión del módulo tanto en arranque como final, pero un enlace aunque inestable en el proceso de prueba pero genera un comportamiento más idóneo para la regresión del modelo de control matemático (Ec.3), lo que podemos verificar en la Figura 10.

\section{Gráfica prueba de arranque nivel 3}

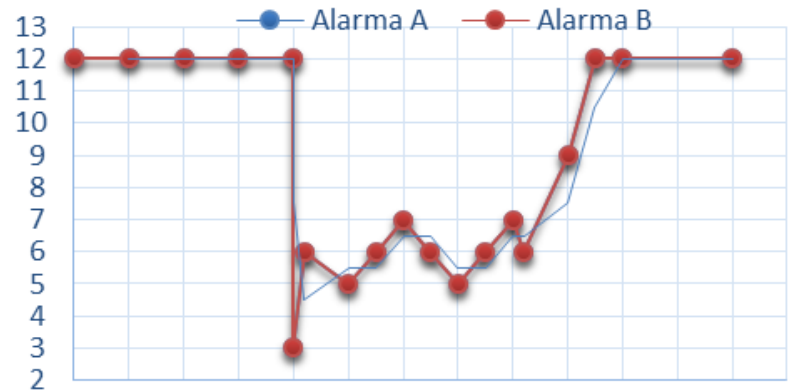

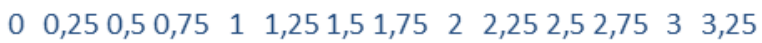

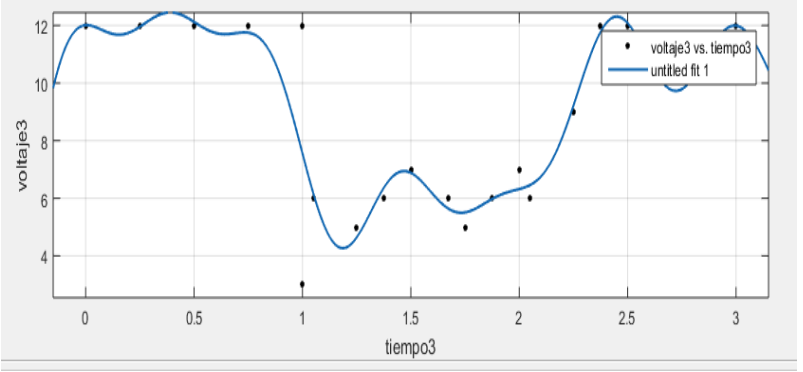

Figura 10. Gráfica prueba de arranque nivel 3

$$
\begin{aligned}
f(t)=a 1 * \sin & (b 1 * t+c 1)+a 2 * \sin (b 2 * t \\
& +c 2)+a 3 * \sin (b 3 * t+c 3) \\
& +a 4 * \sin (b 4 * t+c 4)+a 5 \\
& * \sin (b 5 * t+c 5)+a 6 * \sin (b 6 \\
& * t+c 6)
\end{aligned}
$$

Valores de ajuste: SSE: $42.06, \mathbf{R}^{\mathbf{2}} \mathbf{0} 0.7771=77.71 \%, \mathbf{R M S E}$ : 6.486

Tabla 5. Coeficientes reemplazo ecuación prueba arranque 3

\begin{tabular}{ccccccc}
\hline Coeficientes & $\mathbf{1}$ & $\mathbf{2}$ & $\mathbf{3}$ & $\mathbf{4}$ & $\mathbf{5}$ & $\mathbf{6}$ \\
\hline $\mathrm{a}$ & 14.44 & 10.09 & 1.132 & 4.262 & 0.7789 & 3.047 \\
$\mathrm{~b}$ & 1.005 & 2.067 & 3.262 & 7.652 & 12.16 & 7.605 \\
$\mathrm{c}$ & -0.011 & 1.415 & 2.148 & 2.614 & 3.277 & -6.546 \\
\hline
\end{tabular}

\section{Ensayo condición de arranque nivel 4}

La alarma A como resultado en este ensayo se obtuvo que ésta sale de la función de anti asalto al ejecutarse las pruebas; mientras que la alarma B se mantiene en la función de anti asalto. En la Figura 11 se observa el mismo comportamiento que en la Figura 9, con la diferencia que la caída de tensión es mayor, utilizando por ende el mismo análisis.

El factor de error de la suma de cuadrados indica que los factores de cambio de curva en los puntos de giro son más estables en prueba de arranque 4 , con un factor de error de cuadrados mayor que es de $45.82 \%$ y una aproximación de
$75.48 \%$, generan un comportamiento del proceso en si es mucho más estable en la fase de prueba mas no en su inicio y arranque debido a las condiciones de tensión, generando ese aumento error por el cambio de tensión del módulo tanto en arranque como final, pero un enlace más estable en su proceso de prueba, lo que podemos verificar en la Figura 11 y el modelo matemático (Ec. 4) un acoplamiento estable.
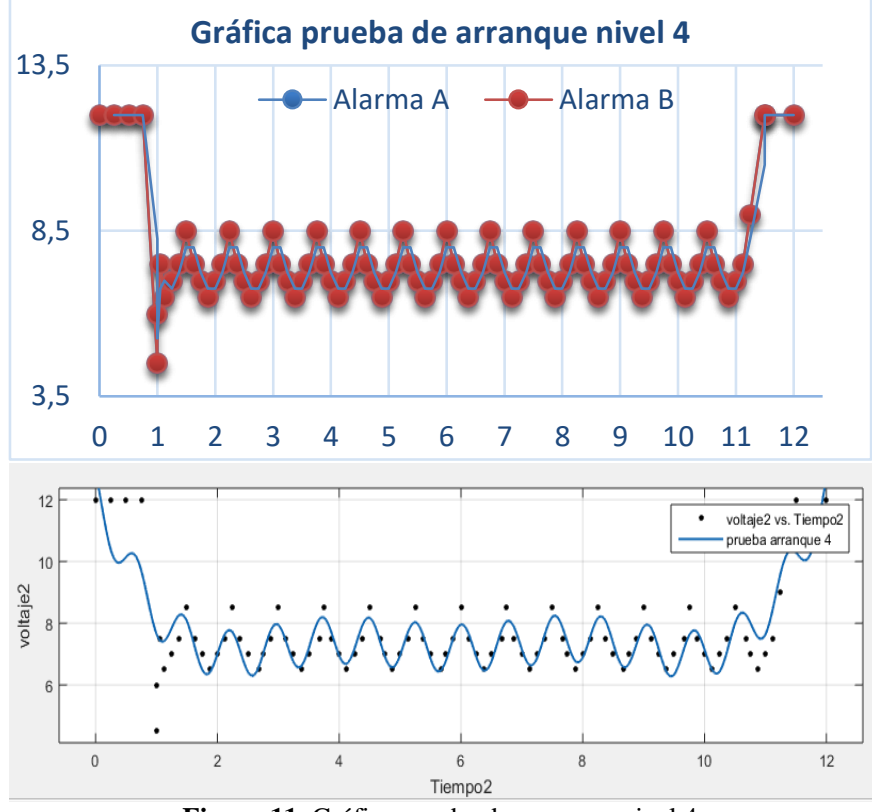

Figura 11. Gráfica prueba de arranque nivel 4

$$
\begin{gathered}
f(t)=a 1 * \sin (b 1 * t+c 1)+a 2 * \sin (b 2 * t \\
+c 2)+a 3 * \sin (b 3 * t+c 3) \\
+a 4 * \sin (b 4 * t+c 4)+a 5 \\
\quad * \sin (b 5 * t+c 5)
\end{gathered}
$$

Valores de ajuste: SSE: $45.82, \mathbf{R}^{2}: 0.7548=75.48 \%, \mathbf{R M S E}$ : 0.7714

Tabla 6. Coeficientes reemplazo ecuación prueba arranque 4

\begin{tabular}{cccccc}
\hline Coeficiente & $\mathbf{1}$ & $\mathbf{2}$ & $\mathbf{3}$ & $\mathbf{4}$ & $\mathbf{5}$ \\
\hline $\mathrm{a}$ & 24.56 & 38 & 31.16 & 10.53 & -0.7752 \\
$\mathrm{~b}$ & 0.3029 & 0.5159 & 0.7039 & 0.8682 & 8.234 \\
$\mathrm{c}$ & -0.196 & 1.675 & 3.688 & 5.845 & -25.94 \\
\hline
\end{tabular}

\section{CONCLUSIONES}

Es importante comprender las diferentes condiciones eléctricas que se realizan para evaluar los dispositivos electrónicos, las condiciones que se han seleccionado para la análisis de estas dos alarmas automotrices se relacionan a condiciones más comunes que puede aparecer en sistema eléctrico del vehículo y como estas pueden interferir en la protección del vehículo ante un robo.

La selección de la función de anti asalto de la alarma automotriz presenta la mayor cantidad de periféricos activados, además de entrar en un laso de programación que solo puede ser desactivado mediante el botón valet o código de desactivación, lo que permite al evaluador poder determinar la valoración que menciona la norma y el criterio del resultado pueda sustentarse en relación a la permanencia o salida de la función durante cada ensayo. 
El cambio de la tensión generan que el comportamiento de los modules se altere conforme se activa o desactiva la prueba, generando un modelo matemático estable a la dispersión de datos obtenidos, que con errores y aproximaciones medias, se analiza que su estabilidad es más alta en el centro de las figuras, generando así una mayor veracidad a la ecuación de control.

Mientras mayor la caída de tensión y la exigencia de la prueba la estabilidad del módulo es menor, aunque su comportamiento en el proceso de funcionamiento en sí, se mantiene estable aunque se vea afectado notablemente su arranque y cierre como se puede apreciar en los esquemas de control de las figuras.

En cuanto a la prueba de caída momentánea de tensión amabas alarma se mantienes en la condición de anti asalta, tomando relación que el tiempo de la ciada del valor de tensión es el mismo pero el valor de tensión mínimo es diferente.

En el ensayo de reseteo ante la caída de tensión la Alarma A durante el tiempo de ejecución de la prueba se mantiene funcional en la condición de anti asalto pero al decrecer a valores inferiores al voltaje mínimo de funcionamiento ésta pierde la condición programada y se vuelve vulnerable en cuanto a su programación o ejecución en la condición de anti asalto. La alarma B a pesar de llegar a valores de tensión mínimos de funcionamiento al terminar la prueba no pierde la condición programada de anti asalto.

En relación a la condición de arranque, en sus 4 niveles la Alarma A pierde la condición de anti asalto como resultado de la variación de tensión que se produce en la alimentación de energía hacia el módulo electrónico. Mientras tanto en la Alarma B, el módulo de alarma se mantiene en la condición de anti asalto durante y después de la ejecución de la prueba. La tensión inicial que estable la norma para esta prueba es de 12 voltios.

\section{REFERENCIAS}

Alonso J.M. (2014).Sistemas de seguridad y confortabilidad, España, Editorial Paraninfo. Pp 105 - 116

Antoni Joan, Barrera Oscar (2011).Sistemas eléctricos, de seguridad y confortabilidad, España, Editorial Paraninfo

Daniels Jeff. (2010). Tecnología del auto moderno, España, Editorial CEAC

Lema, J.,\& Pancha, J. (2016). Diseño y construcción de un módulo electrónico de alarma automotriz evaluada mediante ensayos establecidos en la norma ISO 16750-2(tesis de maestría) Escuela Politécnica Nacional, Quito, Ecuador.

\section{BIOGRAFÍAS}

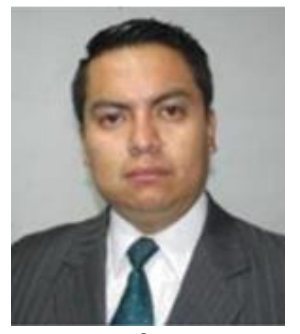

Johnny Pancha. Máster en Sistemas Automotrices por la Escuela Politécnica Nacional; Ingeniero en Mecánica Automotriz por la Escuela Politécnica Javeriana del Ecuador. Tiene experiencia en el área de mantenimiento y reparación de vehículos a gasolina. Se desempeña como profesor y capacitador en el área de Electricidad Automotriz e Inyección Electrónica. Actualmente labora como docente en Escuela Superior Politécnica de Chimborazo como docente en la Escuela de Ingeniería Automotriz.

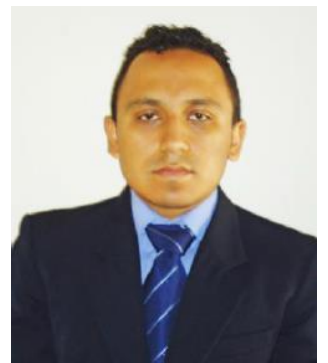

Jorge Lema Loja. Ingeniero en Mecánica Automotriz por la Escuela Politécnica Javeriana del Ecuador, Máster en Sistemas Automotrices por la Escuela Politécnica Nacional. Tiene experiencia en el área de mantenimiento y reparación en vehículos a diésel. Se desempeña como docente universitario en las áreas de los Sistemas de Inyección Electrónica y matemáticas avanzadas para ingeniería. En la actualidad trabaja como docente en la Universidad Tecnológica Indoamérica.

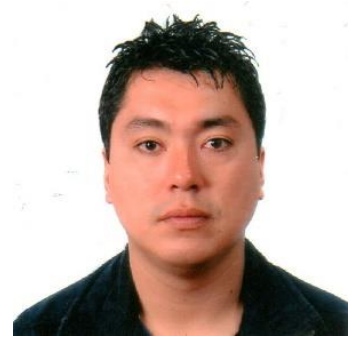

Vicente Rojas. Máster en Sistemas Automotrices por la Escuela Politécnica Nacional (2016); Ingeniero Mecánico Automotriz por la Universidad Politécnica Salesiana (2012), donde fue ayudante de cátedra desde 2008. Fue subgerente general y estructuralista en constructora de estructuras metálicas Efesto. Cuenta con una amplia experiencia en el área automotriz y nuevas tecnologías. Actualmente labora como docente investigador en la Escuela Superior Politécnica de Chimborazo en la Escuela de Ingeniería Automotriz.

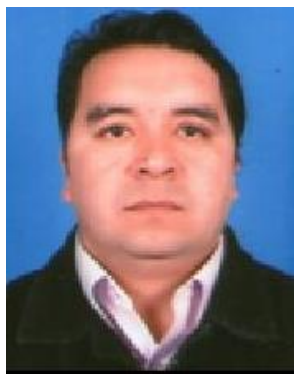

Gerardo Arteaga Rodríguez. Subcoordinador de la Facultad de Ingenierías y Tecnologías de la Información y Comunicación de la Universidad Tecnológica Indoamérica. Ingeniero Mecánico por la Escuela Politécnica Nacional, Master en Sistemas Automotrices por la Escuela Politécnica Nacional. Catedrático y directivo universitario por más de 15 años y hasta la actualidad. Gerente General de la empresa Motor Mundo. 